\title{
Simulation of the acceleration of relativistic electrons in the inner magnetosphere using RCM-VERB coupled codes
}

\author{
D. A. Subbotin, ${ }^{1}$ Y. Y. Shprits, ${ }^{1,2}$ M. Gkioulidou, ${ }^{1}$ L. R. Lyons, ${ }^{1}$ B. Ni, ${ }^{1}$ V. G. Merkin,,${ }^{3,4}$ \\ F. R. Toffoletto, ${ }^{5}$ R. M. Thorne, ${ }^{1}$ R.B. Horne, ${ }^{6}$ and M. K. Hudson ${ }^{7}$ \\ Received 6 December 2010; revised 9 March 2011; accepted 18 May 2011; published 6 August 2011.
}

[1] Radiation belt dynamics have been modeled by the modified Fokker-Planck diffusion equation with sources from the low-energy plasma sheet population and losses to the atmosphere and magnetopause. We perform a coupled simulation of the Rice Convection Model (RCM) and Versatile Electron Radiation Belt (VERB) code. The RCM models magnetospheric convection and provides a low-energy electron seed population for the VERB diffusion code simulations of the Earth's radiation belts. VERB simulations are driven by the realistic time-dependent electron seed population and by the $K p$ index, which is used to specify rates of diffusion by ultralow frequency (ULF) and very low frequency wave activity and, therefore, diffusion processes. Radial diffusion is produced by ULF waves, while pitch angle and energy diffusion are produced by chorus waves outside the plasmasphere and by hiss waves inside the plasmasphere. The results of the simulation indicate that storm time enhanced magnetospheric convection combined with radial diffusion can bring electrons with tens of $\mathrm{keV}$ energy close to the Earth and can affect electron fluxes at 3-4 $R_{E}$. These electrons can be further accelerated locally by chorus waves to $\mathrm{MeV}$ energies. Furthermore, outward radial diffusion smooths out the peak of the high-energy fluxes and produces $\mathrm{MeV}$ electron enhancement around geosynchronous orbit (6-7 $\left.R_{E}\right)$ despite the absence of local electron acceleration in that region. Our coupled simulations indicate that local acceleration in the inner magnetosphere may be a dominant source of relativistic electrons that reach geosynchronous orbit.

Citation: Subbotin, D. A., Y. Y. Shprits, M. Gkioulidou, L. R. Lyons, B. Ni, V. G. Merkin, F. R. Toffoletto, R. M. Thorne, R. Horne, and M. K. Hudson (2011), Simulation of the acceleration of relativistic electrons in the inner magnetosphere using RCM-VERB coupled codes, J. Geophys. Res., 116, A08211, doi:10.1029/2010JA016350.

\section{Introduction}

[2] Earth's radiation belts, discovered by the Explorer 1 mission [Van Allen, 1959], consist of protons and electrons that are trapped by the Earth's magnetic field. The inner electron belt is located at an approximate distance of 1 to 2 Earth radii and is relatively stable, while the outer belt is

\footnotetext{
${ }^{1}$ Department of Atmospheric and Oceanic Sciences, University of California, Los Angeles, California, USA.

${ }^{2}$ Institute of Geophysics and Planetary Physics, University of California, Los Angeles, California, USA. USA.

${ }^{3}$ Center for Space Physics, Boston University, Boston, Massachusetts,

${ }^{4}$ Now at Johns Hopkins University Applied Physics Laboratory, Laurel, Maryland, USA.

${ }^{5}$ Department of Space Physics and Astronomy, William Marsh Rice University, Houston, Texas, USA.

${ }^{6}$ British Antarctic Survey, Natural Environment Research Council, Cambridge, UK.

${ }^{7}$ Department of Physics and Astronomy, Dartmouth College, Hanover, New Hampshire, USA.

Copyright 2011 by the American Geophysical Union. 0148-0227/11/2010JA016350
}

located between approximately 4 and 7 Earth radii [Van Allen and Frank, 1959; Vernov and Chudakov, 1960] and is highly dynamic [Rothwell and McIlwain, 1960; Craven, 1966]. The electron radiation belts consist of highly energetic particles ( $\mathrm{MeV}$ and higher) and can be hazardous to satellite electronics [Baker et al., 1996]. The energetic particles produce charge buildup on various spacecraft materials and eventually cause discharges, damaging the electronics. Since shielding spacecraft subsystems is expensive and not always possible, the outer radiation belt can cause serious problems for satellites operating at all orbits, including geosynchronous orbit, which has a large number of satellites [e.g., Lanzerotti, 2001]. Severe geomagnetic storms also increase the risk of damage to satellites and can lead to satellite failure, especially at geosynchronous orbit [e.g., Odenwald et al., 2006].

[3] Earthward magnetospheric convection brings particles from the tail region to the plasma sheet and can energize electrons by hundreds of $\mathrm{keV}$ [e.g., Lyons, 1984], providing a seed population for radiation belts [e.g., Baker and Stone, 1978; Elkington et al., 2004]. These hundred keV electrons, convected into the region of the radiation belts, are further affected by plasma waves with ultralow frequency (ULF; 
$1.7 \mathrm{mHz}$ to $6.7 \mathrm{mHz}$ ), extremely low frequency (ELF; $3 \mathrm{~Hz}$ to $3 \mathrm{kHz}$ ), and very low frequency (VLF; $3 \mathrm{kHz}$ to $30 \mathrm{kHz}$ ) [Jacobs et al., 1964]. In some cases local acceleration can begin at energies near a few $\mathrm{keV}$ due to interactions with upper band chorus waves [Thorne et al., 2010; Ni et al., 2011a, 2011b].

[4] Quasi-linear interactions with plasma waves can be described by the Fokker-Planck diffusion equation in terms of radial, energy (or momentum), and pitch angle diffusion due to resonant wave-particle interactions [Kennel and Engelmann, 1966; Lerche, 1968; Schulz and Lanzerotti, 1974]. Lyons et al. [1971] were first to quantify the diffusion rates using a high plasma density approximation, which later allowed the evaluation of losses due to whistler mode plasmaspheric hiss waves [Lyons et al., 1972] and provided an explanation for the two zone structure of Earth's radiation belts [Lyons and Thorne, 1973]. More recently, a number of codes have been developed for the calculation of quasi-linear diffusion rates without making a high density approximation [e.g., Glauert and Horne, 2005; Albert and Young, 2005; Albert, 2007; Ni et al., 2008; Shprits and $\mathrm{Ni}, 2009]$.

[5] The potential importance of losses due to outward radial diffusion was indicated by several simulation studies, which used time-varying fluxes at the outer boundary for the simulations [Brautigam and Albert, 2000; Miyoshi et al., 2003, 2006]. Shprits et al. [2006a] eliminated adiabatic changes at the boundary by performing radial diffusion simulations with time-varying outer boundary conditions, which were set up at fixed $L^{*}=7$. They also presented observations of depletions of energetic fluxes at different energies, which excludes the possibility for the depletion to be produced by scattering by Electromagnetic Ion Cyclotron (EMIC) waves. The simulations indicate the importance of the magnetopause losses and convective source to radiation belt dynamics. Li et al. [2007] showed that chorus waves can produce a net acceleration in the recovery phase, while EMIC waves assisted by hiss waves in plumes can produce rapid losses of $\mathrm{MeV}$ electrons.

[6] The first solution of the 3-D Fokker-Planck equation was obtained by the Salammbô code [Beutier and Boscher, 1995; Bourdarie et al., 1996, 1997; Boscher et al., 2000]. The simulation included radial diffusion driven by electrostatic and magnetic field fluctuations, cosmic ray albedo decay, losses due to plasmaspheric hiss, and Coulomb collisions. Varotsou et al. [2005] and Horne et al. [2005] added diffusion due to chorus waves to the Salammbô code and showed that chorus waves can efficiently scatter and accelerate electrons during resonant wave-particle interactions with $\mathrm{MeV}$ energy electrons in the radiation belts. Varotsou et al. [2008] and Shprits et al. [2009] also showed that keV energy electrons can be accelerated to $\mathrm{MeV}$ in the outer radiation belt by a combination of radial diffusion and local acceleration.

[7] Albert et al. [2009], Subbotin et al. [2010], and Xiao et al. [2010] solved the 3-D Fokker-Planck equation, including radial diffusion due to ULF waves, and energy, pitch angle, and mixed energy-pitch angle diffusion due to chorus waves. The results indicate that mixed diffusion is of importance for electrons with equatorial pitch angles of less then $30^{\circ}$, while for electrons with higher equatorial pitch angles the difference between simulations that include versus neglect mixed scattering is minor.
[8] Jordanova and Miyoshi [2005], using a convective model with radial diffusion due to the magnetic component of ULF waves and parameterized losses, showed that magnetospheric convection is the dominant injection mechanism for the low-energy electron seed population, while radial diffusion can transport particles with higher energies to lower L shells. Fok et al. [2008] presented a simulation with the Radiation Belt Environment (RBE) model, which includes magnetospheric convection, and electron losses and acceleration due to resonant wave-particle interactions with chorus waves using the Tsyganenko 2004 [Tsyganenko and Sitnov, 2005] magnetic field model and Weimer [Weimer, 2001] electric field model. The magnetic and electric fields were updated every $5 \mathrm{~min}$ and $3 \mathrm{~s}$, respectively. While the RBE model did not include a radial diffusion term explicitly, it accounted for variable convection that is ignored by other radiation belt modeling codes.

[9] Accurate long-term radiation belt simulations require knowledge of the low-energy electron seed population. The electron seed population can be obtained from observations near geosynchronous orbit. However, to obtain real-time forecasting and to understand the underlying physical processes, magnetospheric convective codes should be used to model the low-energy seed population.

[10] In the current study, we describe our first attempt to conduct a coupled simulation of the VERB code [Subbotin and Shprits, 2009; Shprits et al., 2009] and the Rice Convection Model (RCM) [Toffoletto et al., 2003, and references therein]. In this study, we use one-way code coupling with the electron fluxes from RCM being used by the VERB code, which models resonant wave-particle interactions in the radiation belts. We describe the VERB and RCM models and the coupling between them in section 2 . In section 3 , we present the results of the coupled simulation. Based on the calculated results, in section 4 we show that relativistic electrons at geosynchronous orbit may come from the acceleration of energetic electrons at lower L shells by local energy diffusion and subsequent outward radial diffusion. We summarize our work in section 5 .

\section{Model Description}

\subsection{Radiation Belt Simulation With the VERB Code}

[11] The dynamics of relativistic electrons in the radiation belts can be described by the bounce and Magnetic Local Time (MLT)-averaged Fokker-Planck equation [e.g., Schulz and Lanzerotti, 1974; Subbotin and Shprits, 2009]:

$$
\begin{aligned}
\frac{\partial f}{\partial t}= & \left.\left.L^{* 2} \frac{\partial}{\partial L^{*}}\right|_{\mu, J} \frac{1}{L^{* 2}} D_{L^{*} L^{*}} \frac{\partial f}{\partial L^{*}}\right|_{\mu, J}+\left.\frac{1}{p^{2}} \frac{\partial}{\partial p}\right|_{\alpha_{0}, L} \\
& \cdot p^{2}\left(\left.D_{p p} \frac{\partial}{\partial p}\right|_{\alpha_{0}, L} f+\left.D_{p \alpha_{0}} \frac{\partial}{\partial \alpha_{0}}\right|_{p, L} ^{f}\right)+\left.\frac{1}{T\left(\alpha_{0}\right) \sin \left(2 \alpha_{0}\right)} \frac{\partial}{\partial \alpha_{0}}\right|_{p, L} \\
& \cdot T\left(\alpha_{0}\right) \sin \left(2 \alpha_{0}\right)\left(\left.D_{\alpha_{0} \alpha_{0}} \frac{\partial}{\partial \alpha_{0}}\right|_{p, L} f+\left.D_{\alpha_{0} p} \frac{\partial}{\partial p}\right|_{\alpha_{0}, L} ^{f}\right)+\mathcal{S}-\mathcal{L},
\end{aligned}
$$

where $f$ is the electron Phase Space Density (PSD); $\mu$ and $J$ are the first and second adiabatic invariants (defined below); $p$ is the relativistic momentum; $\alpha_{0}$ is the equatorial pitch angle of the particles; $T\left(\alpha_{0}\right)$ is a function related to the 
bounce frequency and can be approximated as [Lenchek et al., 1961]

$$
T\left(\alpha_{0}\right)=1.3802-0.3198\left(\sin \alpha_{0}+\sin ^{1 / 2} \alpha_{0}\right) .
$$

$D_{L^{*} L^{*}}, D_{p p}, D_{p \alpha 0}, D_{\alpha 0 p}$, and $D_{\alpha 0 \alpha 0}$ in equation (1) are the bounce- and MLT-averaged scattering rates (or diffusion coefficients) due to resonant wave-particle interactions. $\mathcal{S}$ represents the convective sources of particles (see section 4 for more details) and $\mathcal{L}$ represents losses inside the loss cone

$$
\mathcal{L}=\frac{f}{\tau},
$$

where $\tau$ is a characteristic lifetime, assumed to be infinite outside the loss cone and equal to a quarter bounce period inside the loss cone. $L$ is the radial distance from the center of the Earth to the equatorial footprint of the magnetic field line; $L^{*}$ is equal to the distance $L$ if the magnetic field is adiabatically relaxed to a dipole field. $L^{*}$ is inversely proportional to the third adiabatic invariant $\Phi$ as

$$
L^{*}=(2 \pi M) /\left(\Phi R_{E}\right)
$$

where $M$ is the magnetic moment of the Earth's dipole field; $R_{E}$ is the Earth's radius. The $\mu, J$, and $\Phi$ adiabatic invariants may be expressed as

$$
\begin{aligned}
& \mu=\frac{p_{\perp}^{2}}{2 m_{0} B} \\
& J=\int_{\text {bounce }} p_{\|} d s \\
& \Phi=\oint_{\text {drift }} B d S .
\end{aligned}
$$

Relativistic momentum can be related to energy as $E=$ $\sqrt{p^{2} c^{2}+m^{2} c^{4}}-m c^{2}$ and the momentum diffusion is often referred to as energy diffusion.

[12] The first term on the right-hand side of equation (1) describes the radial diffusion of PSD in $L^{*}$, which does not violate the first and second adiabatic invariants $\mu$ and $J$ [Schulz and Lanzerotti, 1974]. The calculation over $L^{*}$ space allows us to exclude PSD variation due to slow changes of the magnetic field. The slow changes of the magnetic field produce adiabatic motion of the particles and do not change $L^{*}$. For the VERB code results to be compared with satellites observations, the $L^{*}\left(L, \alpha_{0}\right)$ values need to be computed along the satellite path. After that, observed and calculated PSD at the same $L^{*}$ should be compared.

[13] The parameterization for the radial diffusion coefficient due to magnetic field fluctuations is adopted from Brautigam and Albert [2000]:

$$
D_{L^{*} L^{*}}=10^{0.056 K p-9.325} L^{10}
$$

Radial diffusion due to electric field fluctuations is much less effective for the outer radiation belt [Brautigam and Albert, 2000] and is thus assumed to be negligible in the current study.

[14] The last four terms of equation (1) define local processes: momentum diffusion with diffusion coefficient $D_{p p}$, pitch angle diffusion with diffusion coefficient $D_{\alpha 0 \alpha 0}$, and mixed diffusion with diffusion coefficients $D_{\alpha 0} \equiv D_{\alpha 0 p}$. Mixed radial-energy and radial-pitch angle scattering are generally considered to be less effective for radial transport as compared to radial diffusion [Falthamm and Walt, 1969; Roederer and Schulz, 1969, 1971], and the diffusion coefficient is likely to be lower than the local diffusion coefficients [Schulz, 1972]. Therefore, to simplify calculations in this initial study, the mixed radial-energy and radial-pitch angle diffusion terms are omitted.

[15] Mixed energy-pitch angle diffusion was recently studied as a 2-D problem [Albert and Young, 2005; Tao et al., 2008, 2009; Xiao et al., 2009] and as a 3-D problem [Albert et al., 2009; Subbotin et al., 2010; Xiao et al., 2010]. The mixed diffusion was shown to be most important for the lower equatorial pitch angles, while affecting the equatorially mirroring particles to a lesser degree. In our simulation, we focus on equatorially mirroring particles and the effects of mixed diffusion are assumed to be small compared to other potential sources of uncertainty, i.e., uncertainty in the wave latitudinal distribution and density model [Shprits et al., 2006b], uncertainty in the spectral property of waves, and inaccuracy due to the assumption of an isotropic pitch angle distribution in the RCM code (see Appendix A).

[16] The local diffusion coefficients $D_{p p}, D_{\alpha_{0} \alpha_{0}}$, and $D_{p \alpha}$ in equation (1) should be computed and used in the real magnetic field geometry. For this initial study we assume a dipole field for the computation of local diffusion coefficients. By using a dipole field for the local scattering computation, the Fokker-Planck equation can simply be MLT averaged, which greatly simplifies the simulations. A more accurate solution should be MLT dependent and use scattering coefficients computed in the realistic magnetic field [e.g., Orlova and Shprits, 2010].

[17] In this study, we assume local diffusion due to chorus waves outside the plasmasphere and due to hiss waves inside the plasmasphere. The assumed wave parameters are similar to those of Shprits et al. [2009] that are listed in Table 1.The corresponding diffusion rates are computed for resonance orders up to $5(n \leq|5|)$ using the Full Diffusion Code (FDC) [Ni et al., 2008; Shprits and Ni, 2009]. Figure 1 shows the resulting diffusion rates in days ${ }^{-1}$, computed for $L^{*}=4.5$, as a function of energy and equatorial pitch angle. Figures 1a and 1b show the pitch angle and normalized momentum diffusion rates due to resonant wave-particle interactions with dayside chorus waves. Figures 1c and 1d show the pitch angle and normalized momentum diffusion rates due to nightside chorus waves, and Figures 1e and 1f show diffusion rates due to plasmaspheric hiss. For dayside chorus waves, pitch angle diffusion rates at the edge of the loss cone are greater than energy diffusion rates, therefore dayside chorus waves mostly produce losses [Shprits et al., 2007a]. Similarly, energy diffusion rates due to nightside chorus waves are greater than pitch angle diffusion rates for $1 \mathrm{MeV}$ particles, so nightside chorus waves mainly energize of $\sim \mathrm{MeV}$ electrons [ $\mathrm{Li}$ et al., 2007]. Plasmaspheric hiss is responsible for losses inside the plasmasphere and can produce only a negligible energization of electrons.

[18] We use two grids for the simulation: the grid for radial diffusion is constructed to be orthogonal in $\mu, J, L^{*}$ coordinates, and the grid for local diffusion is orthogonal in $\left(p, \alpha_{0}\right)$ for each $L^{*}$. For more details about the creation of 
Table 1. Wave Parameters Used for the Diffusion Coefficient Computation

\begin{tabular}{|c|c|c|c|c|c|c|}
\hline Type of Wave & $B_{w}(\mathrm{pT})$ & $\lambda_{\max }$ & Density Model & $\begin{array}{l}\text { Percent } \\
\text { MLT }\end{array}$ & Wave Spectral Properties & $\begin{array}{l}\text { Distribution in } \\
\text { Wave Normal }\end{array}$ \\
\hline Chorus day & $\begin{aligned} & 10^{0.75+0.04 \lambda}\left(2 \times 10^{0.73+0.91 K p}\right)^{0.5} / 57.6 \\
& \text { for } K p \leq 2+; \\
& 10^{0.75+0.04 \lambda}\left(2 \times 10^{2.5+0.18 K p}\right)^{0.5} / 57.6 \\
& \text { for } 2+<K p<6\end{aligned}$ & 35 & [Sheeley et al., 2001] & 25 & $\begin{array}{c}\omega_{m} / \Omega_{e}=0.2, \delta \omega / \Omega_{e}=0.1 \\
\omega_{u c} / \Omega_{e}=0.3, \omega_{l c} / \Omega_{e}=0.1 .\end{array}$ & $\begin{array}{l}\theta_{m}=0^{\circ}, \delta \theta=30^{\circ}, \\
\theta_{u c}=45^{\circ}, \theta_{l c}=0^{\circ}\end{array}$ \\
\hline Chorus night & $\begin{array}{c}50\left(2 \times 10^{0.73+0.91 K p}\right)^{0.5} / 57.6 \\
\text { for } K p \leq 2+; \\
50\left(2 \times 10^{2.5+0.18 K p}\right)^{0.5} / 57.6 \\
\text { for } 2+<K p \leq 6\end{array}$ & 15 & [Sheeley et al., 2001] & 25 & $\begin{array}{l}\omega_{m} / \Omega_{e}=0.35, \delta \omega / \Omega_{e}=0.15 \\
\omega_{u c} / \Omega_{e}=0.65, \omega_{l c} / \Omega_{e}=0.05\end{array}$ & $\begin{array}{l}\theta_{m}=0^{\circ}, \delta \theta=30^{\circ}, \\
\theta_{u c}=45^{\circ}, \theta_{l c}=0^{\circ}\end{array}$ \\
\hline $\begin{array}{l}\text { Plasma- spheric } \\
\text { hiss }\end{array}$ & $30 \times K p / 4$ & 40 & $\begin{array}{l}\text { [Carpenter and } \\
\text { Anderson, 1992] }\end{array}$ & 60 & $\begin{aligned} f_{m} & =550 \mathrm{~Hz}, \delta f=300 \mathrm{~Hz} \\
f_{u c} & =2000 \mathrm{~Hz}, f_{l c}=100 \mathrm{~Hz}\end{aligned}$ & $\begin{array}{l}\theta_{m}=0^{\circ}, \delta \theta=20^{\circ}, \\
\theta_{u c}=30^{\circ}, \theta_{l c}=0^{\circ} .\end{array}$ \\
\hline
\end{tabular}

the numerical grid and the VERB code see Subbotin and Shprits [2009].

[19] Following previous studies [Subbotin and Shprits, 2009; Shprits et al., 2009], the initial condition for the simulation is obtained by solving a steady state radial diffusion equation for each energy and pitch angle with parameterized electron lifetimes. The radial diffusion coefficient was calculated using equation (6) with the $K p$ index from the beginning of 21 April. The radial diffusion steady state solution provides a reasonable radial distribution of electron fluxes [e.g., Shprits and Thorne, 2004].

[20] To solve equation (1) and perform the 3-D radiation belt simulation, we need to specify the simulation domain and six boundary conditions, two for each variable in the equation. Inside the loss cone, we set PSD to zero $(P S D=0)$,

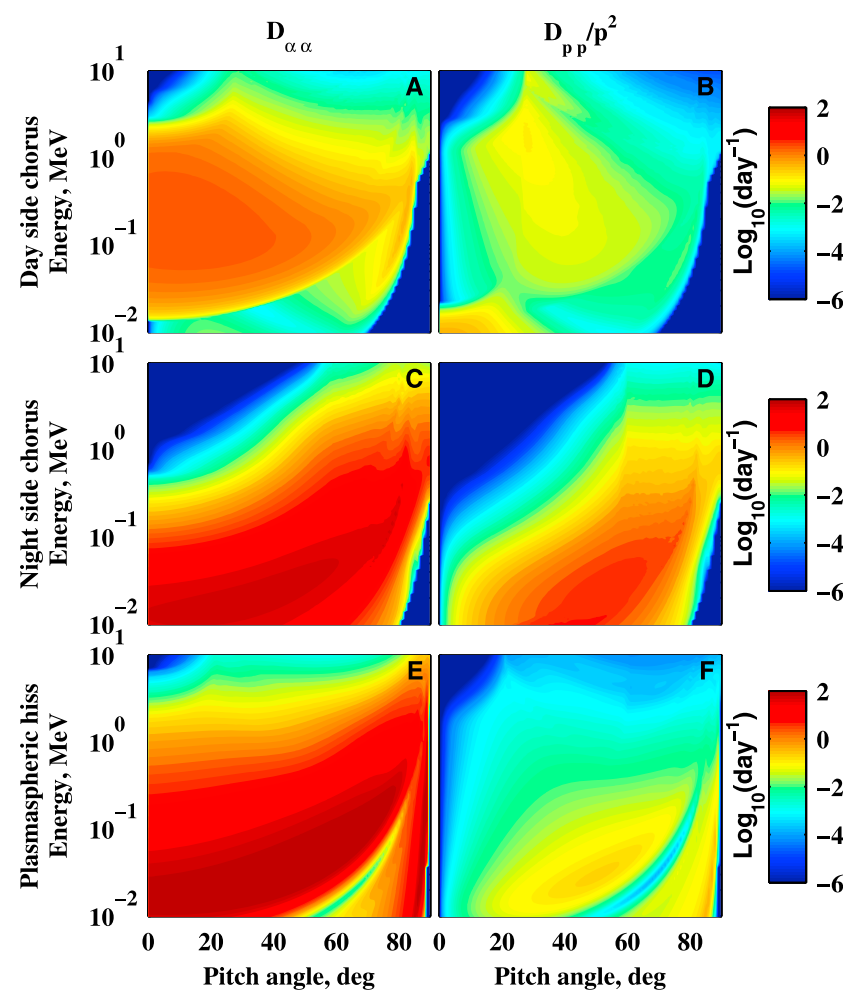

Figure 1. (a) Pitch angle and (b) normalized energy diffusion rates due to dayside chorus waves computed at $L^{*}=4.5$ color coded as $\log _{10}\left(\right.$ day $\left.^{-1}\right)$ as a function of energy and equatorial pitch angle. (c and d) Diffusion rates due to nightside chorus and (e and f) plasmaspheric hiss waves. assuming weak diffusion [Kennel and Petschek, 1966]. For equatorially mirroring particles, PSD is set to have a zero gradient $\left(\partial P S D / \partial \alpha_{0}=0\right)$, which represents the observed flat distribution of equatorially mirroring particles [Horne et al., 2003]. On the lower boundary for radial diffusion at $L^{*}=1$, PSD is set to zero at all energies since particles close to the Earth are lost to the atmosphere. A zero PSD condition is also set at the high-energy boundary because of the absence of high-energy $(>10 \mathrm{MeV})$ electrons. PSD at the last two boundaries, i.e., the low-energy boundary and the outer $L^{*}$ boundary, is highly dynamic during storm times and should be inferred from time-dependent satellite observations or from another simulation. Simulations with time-dependent boundary conditions set from radiation belt observations might show a good agreement with observations [Shprits et al., 2006a; Albert et al., 2009], however such simulations do not allow forecasting or nowcasting. In the current work, we use time-dependent results from the RCM code at the outer $L^{*}$ and low-energy computational boundaries. The RCM model is driven by solar wind parameters, so no radiation belt observations are used to drive the simulations, which makes the code useful for predictive simulations. For coupling purposes, the outer radial diffusion boundary is set at $L^{*}=7$ and the energy range at $L^{*}=7$ is set to 0.01 to $10 \mathrm{MeV}$ and varies with $L^{*}$ according to the conservation of the first and second adiabatic invariants [Schulz and Lanzerotti, 1974]. We describe how the outer $L^{*}$ and lowenergy boundaries were chosen in section 2.2. The boundary conditions are summarized in Table 2. The description of the coupling process between the RCM and VERB codes is the main goal of this manuscript.

\subsection{Magnetospheric Convection Computation With the RCM Code and the Coupling Process}

[21] The RCM simulates the convection of protons and electrons in the inner and middle magnetosphere, including the electrodynamic coupling to the ionosphere. The RCM calculates the bounce-averaged magnetic and electric drift

Table 2. Boundary Conditions Used for the VERB Code Simulations

\begin{tabular}{lcl}
\hline Boundary & Condition & Underlying Physical Processes \\
\hline$\alpha_{0}=0^{\circ}$ & $P S D=0$ & empty loss cone \\
$\alpha_{0}=90^{\circ}$ & $\partial(P S D) / \partial \alpha_{0}=0$ & flat pitch angle distribution \\
$L^{*}=1$ & $P S D=0$ & losses to the atmosphere \\
$L^{*}=7$ & $P S D($ time $)$ & coupling with RCM \\
$E=E_{\min }$ & $P S D($ time $)$ & coupling with RCM \\
$E=E_{\max }$ & $P S D=0$ & absence of multi-MeV \\
& & energy electrons \\
\hline
\end{tabular}


of a magnetic flux tube, filled with an isotropic distribution of ions and electrons, using a self-consistently computed electric field and specified magnetic field. In the current study, the Tsyganenko 96 (T96) magnetic field model is used [Tsyganenko, 1995]. For a detailed description of the RCM code, see Toffoletto et al. [2003].

[22] In the current study, the RCM outer boundary conditions are set at $-20 R_{E}$ at midnight, $10 R_{E}$ at noon, and $15 R_{E}$ at dawn and dusk, while the inner boundary is specified at the distance corresponding to $10^{\circ}$ latitude in the ionosphere. The proton and electron energy spectra at the RCM's outer boundary are established as a function of MLT and the interplanetary conditions based on 11 years of Geotail data [Wang et al., 2007; Gkioulidou et al., 2009]. Due to the fact that the majority of the Geotail data is from nonstorm periods, linear extrapolation is currently used to estimate the particle boundary conditions corresponding to storm time interplanetary conditions.

[23] The RCM code provides the near-Earth electron flux distribution that results from magnetospheric convection. The near-Earth low-energy electron fluxes form a seed population for the radiation belts. These fluxes are used as an input for radiation belt modeling by the VERB code.

[24] To illustrate the process of coupling between the RCM and VERB codes, we use the solar wind conditions from the 21 April 2001 magnetic storm (Figure 2). The observed interplanetary conditions are used as inputs for the $\mathrm{RCM}$, that is, for the cross polar cap potential drop that determines the convection strength, for the particle boundary condition (see description above), and for the T96 magnetic field model. The main phase of the storm started on 21 April with a sudden increase of solar wind density (Figure $2 \mathrm{a}$ ) and velocity (Figure $2 \mathrm{~b}$ ), followed by a decrease of the Interplanetary Magnetic Field (IMF) $B_{z}$ (Figure 2c), a decrease in the Dst index and an increase in $K p$ index (Figures $2 \mathrm{~d}$ and 2e). The main phase of the storm lasted for about $0.5-1$ day, during which Dst decreased to $-100 \mathrm{nT}$. The Dst index then increased back to quiet time values during a 1.5 day recovery phase that started at the beginning of the second day of the storm. The storm lasted approximately 2-2.5 days. The combined RCM-VERB simulation of this storm demonstrates how these two codes can be coupled together to provide results of a radiation belt simulation with a time-dependent convective source of lowenergy particles. A quantitative comparison of the results with satellite observations may require more accurate measurements of the magnetic field and will be a subject of future work.

[25] A schematic representation of the coupling process is presented in Figure 3. The equatorial plane of the Earth's magnetosphere is divided into two regions, one where the dominant transport is convection (light shading, further from the Earth) and another where the dominant transport is diffusion (dense shading, closer to the Earth), with a transition region at $L^{*} \approx 7$. In the convection region of the equatorial plane, the motion of the electrons with $\mathrm{keV}$ energies is guided by $E \times B$ magnetospheric drift. When moving toward the Earth and entering into a region of a stronger magnetic field, particles gain energy according to the conservation of the first adiabatic invariant $\mu$. The energy- and charge-dependent gradient and curvature drifts deflect electrons eastward into the region of the ring current
(Figure 3, red convection arrows). On the dayside, lowenergy electrons are lost to the magnetopause, while highenergy electrons make their way around the Earth and may find themselves orbiting on closed trajectories. The circular motion of the high-energy electrons (Figure 3, red circular arrow) trapped by the Earth's magnetic field is further affected by the resonant wave-particle interactions, and is modeled by the VERB code.

[26] To use RCM results as an input for the VERB code, we need to calculate the adiabatic invariants $\left(L^{*}, \mu, J\right)$ on the RCM grid. To find $L^{*}$, we trace the particles' azimuthal drift around the Earth, and infer the total magnetic flux and relate it to $L^{*}$ using equation (5). Since the VERB model is driven by the $K p$ index, and for simplicity, we used the $K p$-dependent Tsyganenko 89c magnetic field model [Tsyganenko, 1989] for these calculations using the Office National d'Études et de Recherche Aerospatiales (ONERA) library [Boscher et al., 2008]. The resulting $L^{*}$ calculated on the RCM grid after 1 day of RCM simulation is shown as an example in Figure 4.

[27] The invariant $\mu$ is computed according to equation (5). Since RCM assumes pitch angle anisotropy, it does not provide a reasonable pitch angle distribution, and we only use electrons with $90^{\circ}$ pitch angles from the RCM results. The invariant $J$ is equal to zero for the equatorially mirroring particles. To represent a realistic pitch angle distribution, we model the equatorial pitch angle distribution assuming a sinusoidal dependence

$$
\operatorname{PSD}\left(\alpha_{0}\right)=\operatorname{PSD}\left(\alpha_{0}=90^{\circ}\right) \cdot \sin \left(\alpha_{0}\right) \text {. }
$$

[28] Figure 5 shows fluxes of equatorially mirroring electrons at midnight as a function of $L^{*}$ and energy after 1 day of RCM simulation and is overplotted by the RCM grid (dotted lines) and the VERB grid (bold solid lines). Energy and pitch angle values are computed using a dipole field model. These are the fluxes a satellite would see if the geomagnetic field would slowly be reduced to a dipole. The fluxes are color coded as $\log _{10}\left(\# / \mathrm{sr} / \mathrm{s} / \mathrm{cm}^{2} / \mathrm{keV}\right)$. The solid green line at $L^{*}=7$ and the solid magenta line at $\mu=11 \mathrm{MeV} / \mathrm{G}$ (energy $\approx 100 \mathrm{keV}$, varies with $L^{*}$ ) represent the interface between the RCM and VERB models. Liu et al. [2003] showed that electrons with energies $\geq 100 \mathrm{keV}$ at $L \sim 3-5$ are affected more by diffusion than by convection. They also showed that a storm time enhancement of 10 to $50 \mathrm{keV}$ energy electrons at $L \sim 3-5$ can be explained by enhanced convection only. While electrons with energies around 10 to $100 \mathrm{keV}$ in the transition region $5 \leq L^{*} \leq 7$ are almost equally affected by both convective and diffusive processes, for the purpose of this initial computing effort, we make a crude approximation by assuming that the electrons above $L^{*}=7$ and the electrons below the low-energy boundary $(\mu<11 \mathrm{MeV} / \mathrm{G})$ are transported by convection only and that the high-energy electron fluxes below $L^{*}=7$ are affected by diffusion process only. $L^{*}=7$ is chosen as a boundary because it's located far enough from the heart of the outer belt, but still is usually a closed drift shell. The invariant $\mu$ should be held constant along the radial diffusion grid lines, since ULF waves do not violate $\mu$ and $J$. Therefore, we need to use a low enough $\mu$ value to resolve $1 \mathrm{MeV}$ energy at low $L^{*}$. The $\mu=11 \mathrm{MeV} / \mathrm{G}$ corresponds to an energy of $10 \mathrm{keV}$ at $L^{*}=7,50 \mathrm{keV}$ at $L^{*}=4$, and $600 \mathrm{keV}$ at $L^{*}=1.5$. We use a 

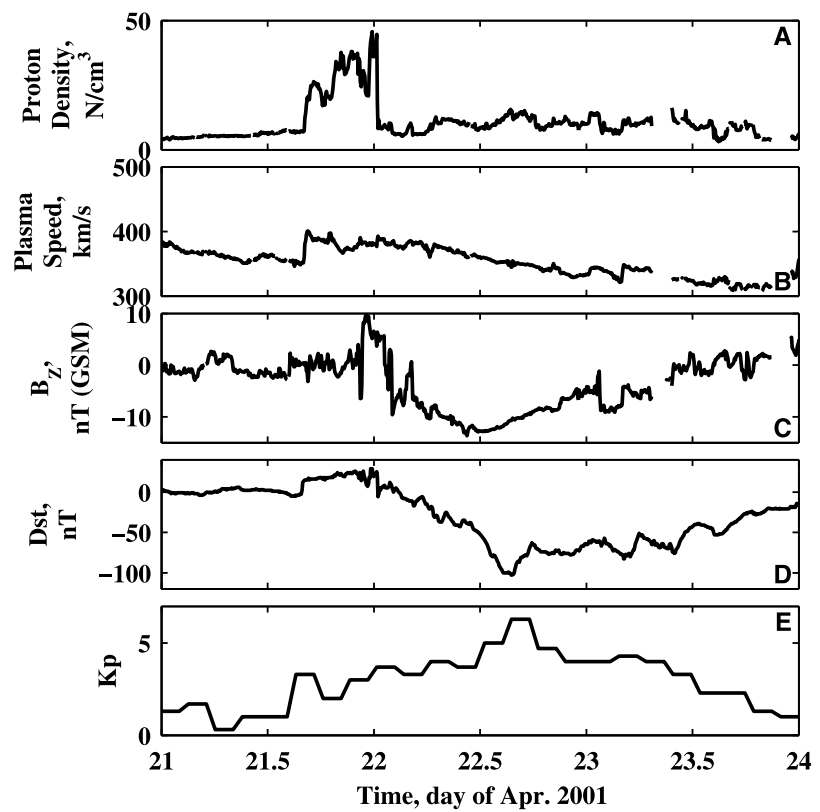

Figure 2. Solar wind and magnetic field parameters for the April 2001 storm. From top to bottom: solar wind proton number density, velocity, $z$ component of the magnetic field, $D s t$ index, and $K p$ index.

RCM-calculated PSD at $L^{*}=7$ for all energies as an outer boundary for the radial diffusion calculation (Figure 5, green line). RCM results are also used at all $L^{*}$ at $\mu=11 \mathrm{MeV} / \mathrm{G}$ as a low-energy boundary for energy diffusion (Figure 5, magenta line).

[29] Since there is currently no MLT dependence in the VERB code, the RCM results are also MLT averaged. Figure 6 compares MLT-averaged electron fluxes of equatorially mirroring particles at geosynchronous orbit during the 3 days of the April 2001 storm computed by the RCM code (color-coded time) with statistical results obtained from previous studies [e.g., Shprits and Thorne, 2004; Shprits et al., 2009] (black dashed line). The black dots show electron fluxes of equatorially mirroring particles, observed

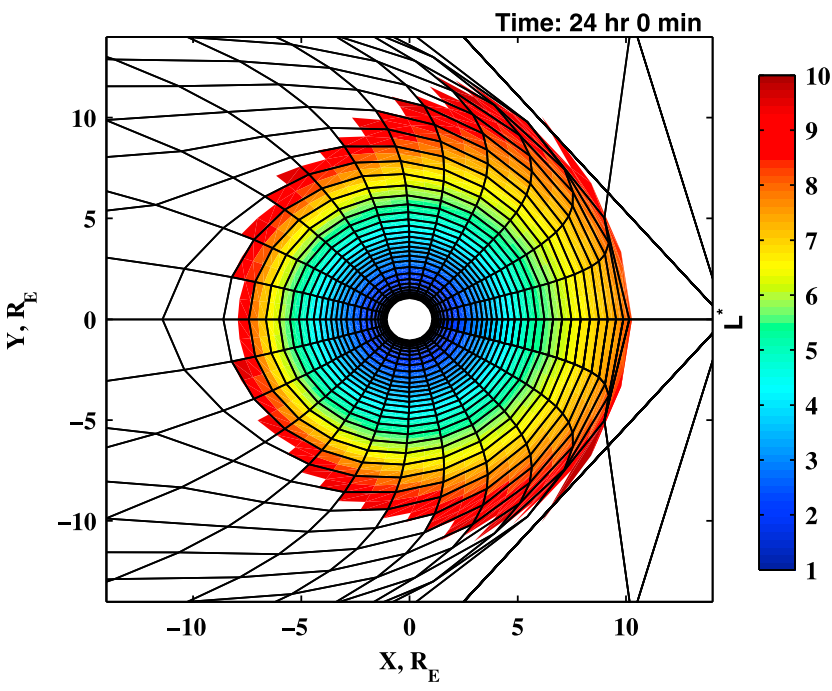

Figure 4. $L^{*}$ computed on the RCM grid after 1 day of RCM simulation (shown as an example). Only every second grid point is plotted for clarity.

on the LANL97A satellite during the years 2000-2003. For each LANL97A satellite location, $L$ was calculated by tracing magnetic field lines to the equatorial plane using the Tsyganenko 1996 geomagnetic field model. The measurements around $L=6.6( \pm 0.1)$ are used, which correspond to crossings of the equatorial plane for a geosynchronous satellite (394 satellite locations were used in total). The spread in observations represents a typical electron flux variation during the 2000-2003 time period. Although fluxes over a broad range of energies can vary by many orders of magnitude from one time interval to another, in general, the RCM fluxes (Figure 6, color-coded lines) are in an agreement with the statistical data.

[30] However, the RCM fluxes are slightly overestimated at low energies and significantly underestimated at high energies, which may suggest that there are missing physical processes. Low-energy electrons are affected by the very rapid losses during strong diffusion on the nightside, leading to the diffuse aurora. These losses are not currently accounted

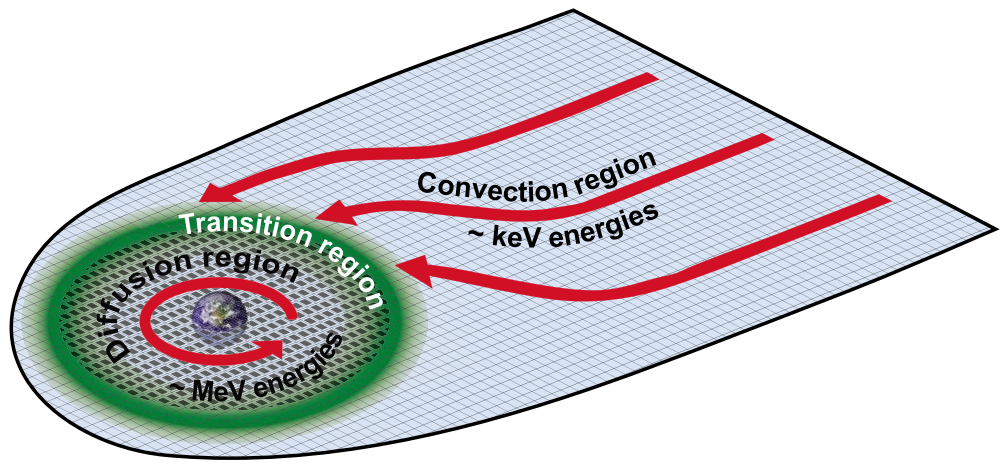

Figure 3. Schematic representation of the RCM-VERB coupling process in the equatorial plane of Earth's magnetosphere: magnetospheric convection, calculated by the RCM code, brings low-energy electrons closer to the Earth, providing a seed population for the radiation belt modeling by the VERB code. A convection-dominated region in the tail (light shading) is separated from the diffusion-dominated region close to the Earth (dense shading) by the transition region around $L^{*} \sim 7$ (denoted by green). 


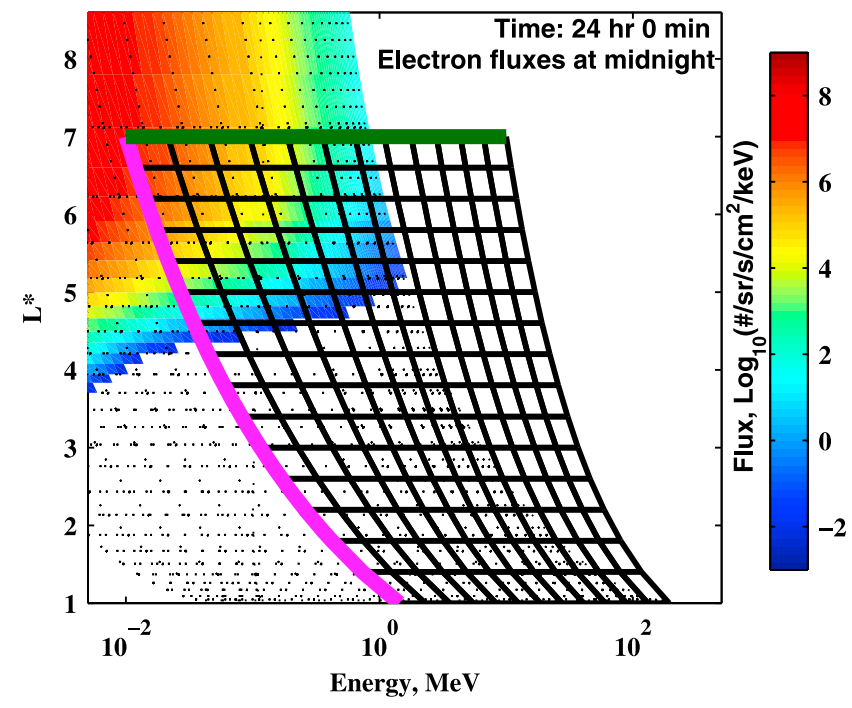

Figure 5. Fluxes at midnight as a function of $L^{*}$ and energy after 24 h of RCM simulation (shown as an example) overplotted by (dotted lines) the RCM grid and (bold solid lines) the VERB grid. Only every second grid point is plotted for clarity. Fluxes are color coded as $\log _{10}\left(\# / \mathrm{sr} / \mathrm{s} / \mathrm{cm}^{2} / \mathrm{keV}\right)$. Green and magenta lines indicate boundary conditions, provided by the RCM code, for the VERB simulations.

for by the RCM simulation. The smaller high-energy flux is probably due to the neglect of local acceleration and radial diffusion in the RCM simulation. The potentially missing sources of high-energy particles are discussed in more detail in section 4 .

\section{Coupled Simulation}

[31] As discussed in section 2.1, three-dimensional radiation belt simulations require six boundary conditions. Four of them can be obtained from physical considerations, i.e., absence of field-aligned particles, absence of electrons at low altitudes due to close proximity to the atmosphere, absence of multi- $\mathrm{MeV}$ energy electrons, and the flat pitch angle distributions at a $90^{\circ}$ equatorial pitch angle. However, knowledge of the time-dependent fluxes at the outer $L^{*}$ and the low-energy boundaries is essential for a time-dependent simulation. In this study, we use RCM modeled fluxes to specify these time-dependent boundary conditions.

[32] In section 3.1, we only use the RCM-calculated PSD for an outer $L^{*}$ boundary (green line on Figure 5) in the VERB code computation and compare the result with a noncoupled simulation that incorporates the statistically averaged data at the outer $L^{*}$ boundary, as was done in previous studies [e.g., Shprits et al., 2009].

[33] In section 3.2, we describe coupling at both lowenergy and outer $L^{*}$ boundaries and present a comparison of the corresponding results.

\subsection{Outer $L^{*}$ Boundary}

[34] Figure 7 shows the results of a 3 day noncoupled simulation of the 21 April 2001 storm with electron flux held constant in time at the outer $L^{*}$ (Figure 7, top) and lowenergy (Figure 7, middle) boundaries. The fluxes at the outer $L^{*}$ boundary are obtained from the time-averaged statistical data, while the fluxes at the low-energy boundary are based on the assumption of balance between convective source and losses. In this simulation, the outer $L^{*}$ and lowenergy boundary conditions are taken from a previous study by Shprits et al. [2009].

[35] Figure 7 (bottom) shows the calculated fluxes of $1 \mathrm{MeV}$ equatorially mirroring electrons. The radiation belt fluxes are relatively low on the first day of the simulation, which represents prestorm conditions. Inward radial diffusion due to increased ULF wave activity during the main phase of the storm (the second day on Figure 7) brings particles to a lower $L^{*} \approx 4$. Such inward motion of the energetic particles' flux is a commonly observed process of slot region refilling [e.g., Thorne et al., 2007]. During the recovery phase (third day on Figure 7), local acceleration due to chorus waves increases and PSD peaks develop at $L^{*}$ $\approx 4$, which is also consistent with the often observed evolution of fluxes during storms. The simulation therefore reproduces the major storm features, i.e., inward flux motion during the main phase of the storm and buildup of fluxes in the recovery phase; however, the simulated flux values are low and the flux enhancement during the recovery phase is moderate.

[36] To provide the VERB code with modeled timevarying values at the outer $L^{*}$ boundary, we introduce RCM-calculated fluxes at $L^{*}=7$. The storm time fluxes, calculated using the outer $L^{*}$ boundary provided by RCM, are shown in Figure 8. The flux variation at $L^{*}=7$ (Figure 8, top) represents the dynamics of the seed population. An increase in fluxes at the beginning of the storm (the end of

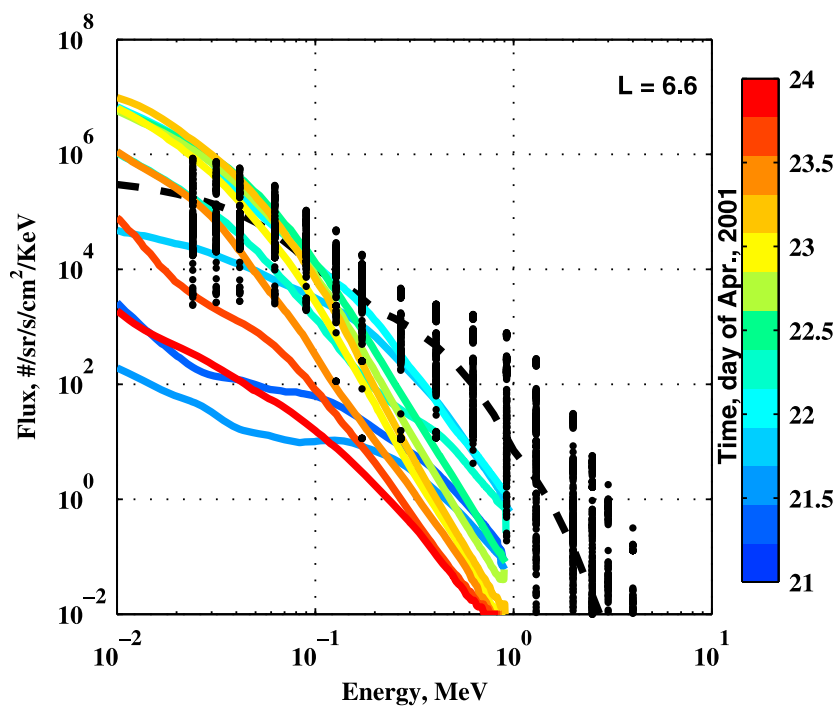

Figure 6. Statistical model for the MLT-averaged equatorially mirroring electron fluxes at geosynchronous orbit [e.g., Shprits and Thorne, 2004; Shprits et al., 2009] used for previous VERB simulations (dashed line) and the RCM simulation with color-coded time (color-coded lines). Black dots show fluxes of equatorially mirroring electrons, observed on the LANL97A satellite during the years 2000 2003 around $L=6.6( \pm 0.1)$ (394 satellite locations were used in total). 


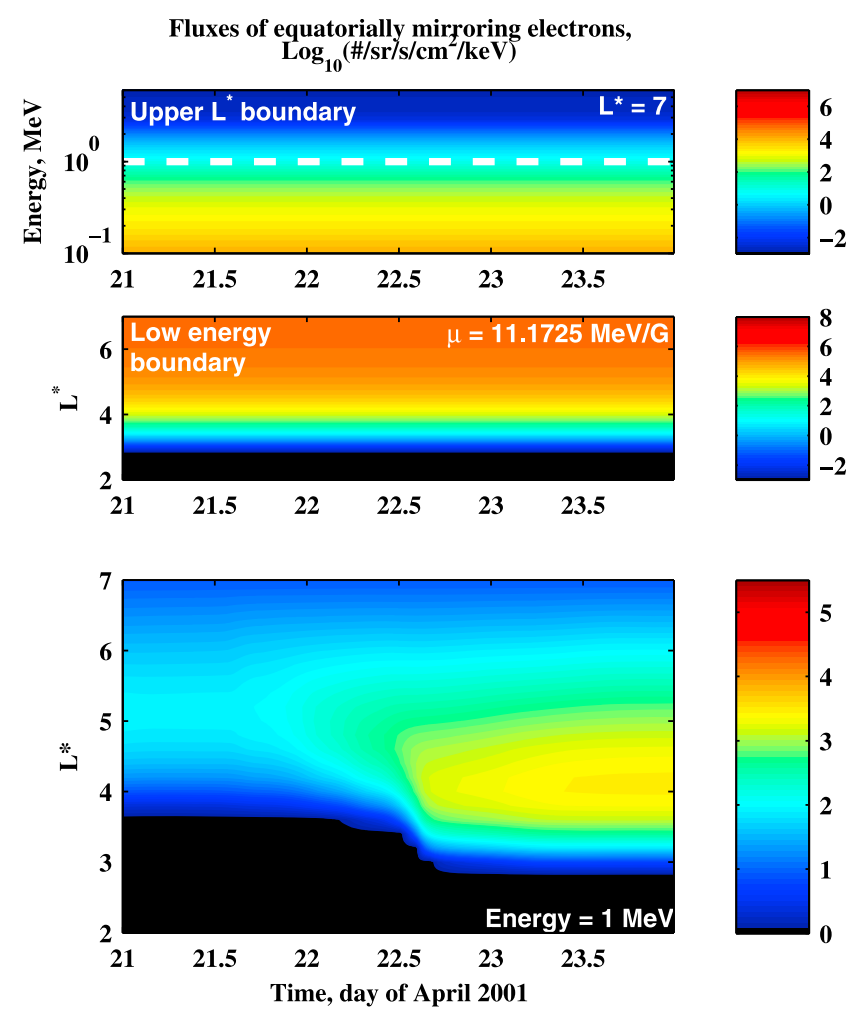

Figure 7. Fluxes of equatorially mirroring electrons after 3 days of a stand-alone VERB simulation, starting on 21 April 2001 , using statistical data at the outer $L^{*}$ boundary and a steady state solution of the radial diffusion equation at the low-energy boundary. (top) Statistical data at $L^{*}=7$ versus time and energy taken from Shprits et al. [2009]. A dashed line is plotted at $1 \mathrm{MeV}$ energy. (middle) Radial diffusion steady state fluxes at the low-energy boundary $(\mu=11$ $\mathrm{MeV} / \mathrm{G}$ ) versus time and $L^{*}$. (bottom) Fluxes of $1 \mathrm{MeV}$ equatorially mirroring electrons calculated by the VERB code plotted versus time and $L^{*}$. Fluxes are color coded as $\log _{10}\left(\# / \mathrm{sr} / \mathrm{s} / \mathrm{cm}^{2} / \mathrm{keV}\right)$.

the first day of the simulation) is followed by a gradual decrease of the fluxes during the next 2 days.

[37] In the simulation presented in Figure 8, we use the same low-energy boundary as in the noncoupled simulation (Figure 7); however, fluxes at the outer $L^{*}$ boundary vary in time according to RCM simulations. The resulting fluxes of the $1 \mathrm{MeV}$ equatorially mirroring electrons are shown in Figure 8 (bottom). Comparing these to the result of the simulation that uses statistically averaged data at the outer $L^{*}$ boundary (Figure 7, bottom), an injection of $1 \mathrm{MeV}$ electrons during the second half of the first day from $L^{*}=7$ is noticeable. These electrons propagate down to $L^{*} \approx 4$ due to radial transport. The overall picture shows a rather typical evolution of radiation belt fluxes. Inward radial motion moves fluxes to the lower $L^{*}$ as well as brings injected electrons from $L^{*}=7$ to $L^{*} \approx 4$ during the main phase. Local acceleration due to chorus waves increases the peak around $L^{*}=4$ during the recovery phase of the storm. However, the maximum value of $\mathrm{MeV}$ fluxes is larger in the simulation with the RCM boundary as compared to the simulation with a constant outer $L^{*}$ boundary as presented in Figure 7. This is the result of the larger fluxes of low- energy particles at $L^{*}=7$. The peak also becomes more pronounced due to both a larger population of low-energy particles and a smaller population of high-energy particles at $L^{*}=7$. The low-energy electrons are transported inward by radial diffusion and accelerated by chorus waves in the recovery phase of the storm.

\subsection{Low-Energy Boundary}

[38] In section 3.1, we assume the low-energy fluxes to be constant in time. However, these fluxes experience dramatic changes during storms and can vary by an order of magnitude [e.g., Jordanova and Miyoshi, 2005; Li et al., 2010]. To include the dynamics of the low-energy population in radiation belt simulations, we incorporate magnetospheric convection dynamics calculated by the RCM code at the low-energy boundary. Figure 9 shows the result of the simulation with RCM fluxes applied to the outer $L^{*}$ boundary (green line on Figure 5) and the low-energy VERB code boundary (magenta line on Figure 5). Figure 9 (top) shows the RCM-calculated fluxes at $L^{*}=7$, which are the same as those shown in Figure 8 (top). Figure 9 (middle) shows the RCM-calculated fluxes at $\mu=11 \mathrm{MeV} / \mathrm{G}$, which are used as the low-energy VERB code boundary. As can be seen in the plot, during the prestorm phase (first half of the first day of the simulation) the magnetospheric convection does not bring particles to the region below $L^{*} \approx 5-6$, while in the main phase of the storm (second day of the

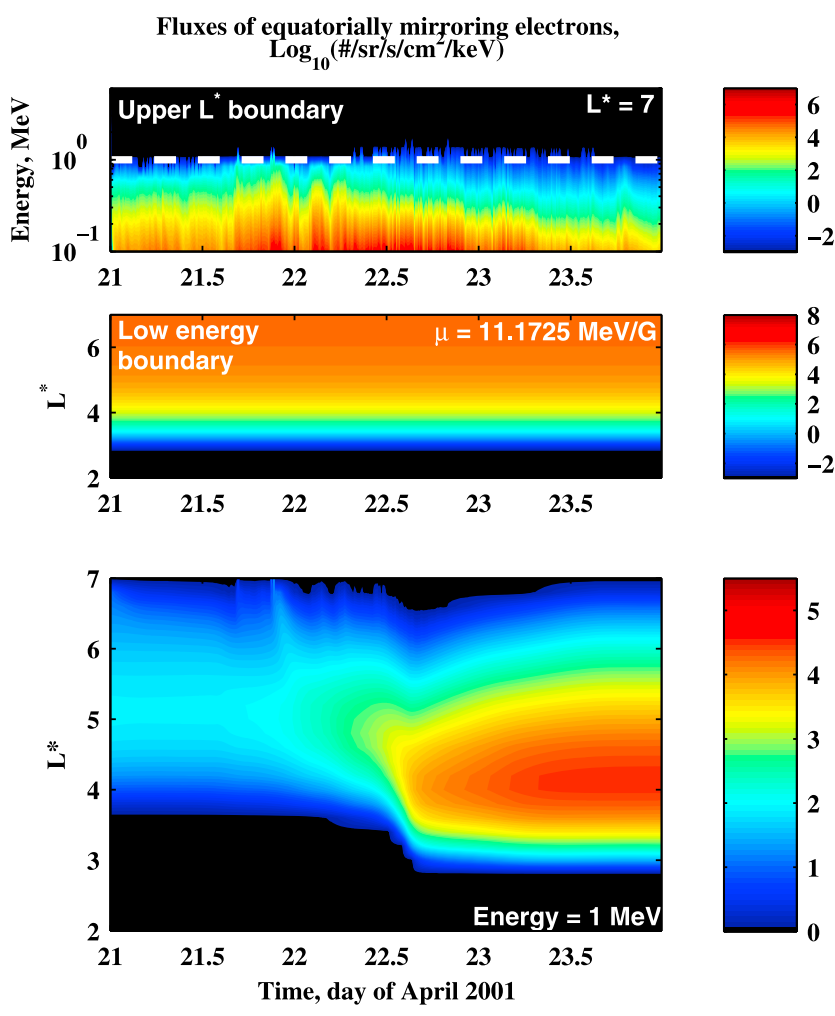

Figure 8. Same as Figure 7 but with outer $L^{*}$ boundary fluxes provided by RCM. (top) RCM-calculated fluxes at $L^{*}=7$. A dashed line is plotted at $1 \mathrm{MeV}$ energy. (middle) Radial diffusion steady state fluxes at the low-energy boundary. (bottom) Fluxes of $1 \mathrm{MeV}$ equatorially mirroring electrons, calculated by the VERB code. Fluxes are color coded as $\log _{10}\left(\# / \mathrm{sr} / \mathrm{s} / \mathrm{cm}^{2} / \mathrm{keV}\right)$. 


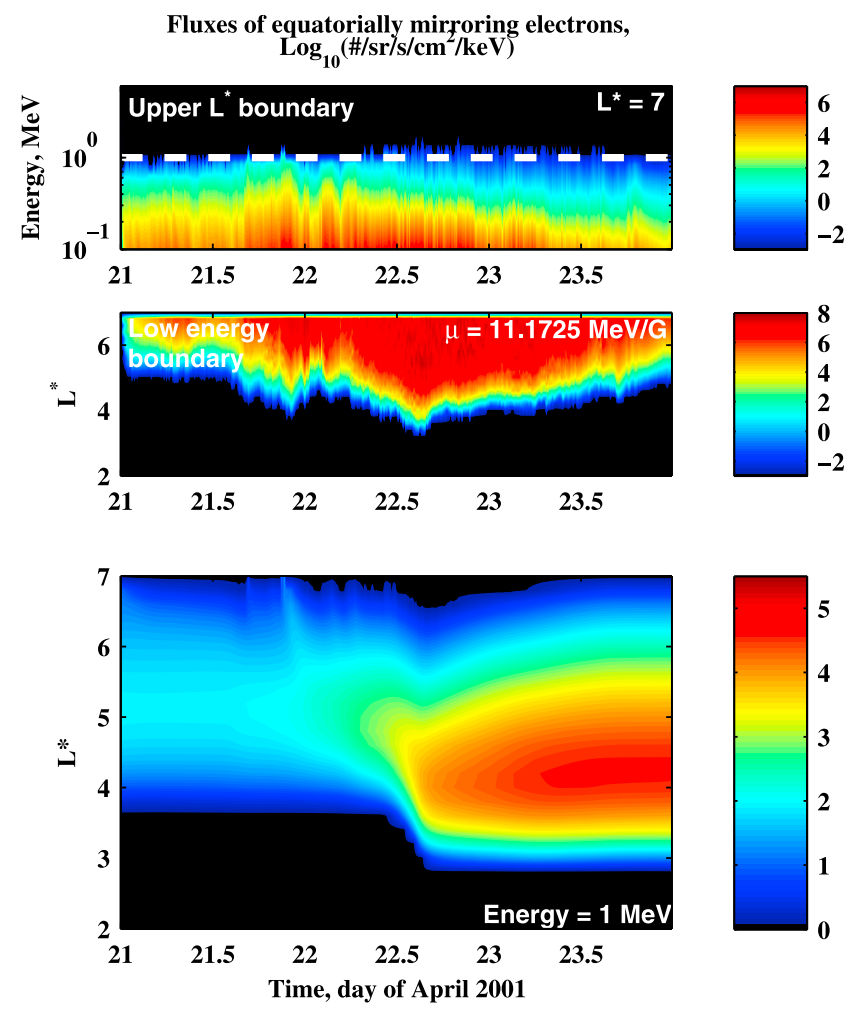

Figure 9. Same as Figure 8 but with RCM-calculated fluxes used at the low-energy boundary. (top) RCM-calculated fluxes at $L^{*}=7$. A dashed line is plotted at $1 \mathrm{MeV}$ energy. (middle) RCM-calculated fluxes at $\mu=11 \mathrm{MeV} / \mathrm{G}$ (lowenergy boundary). (bottom) One $\mathrm{MeV}$ electron fluxes of equatorially mirroring particles, calculated by the VERB code. Fluxes are color coded as $\log _{10}\left(\# / \mathrm{sr} / \mathrm{s} / \mathrm{cm}^{2} / \mathrm{keV}\right)$.

simulation) the low-energy fluxes can be transported earthward to $L^{*} \approx 3-4$.

[39] Despite the significant difference between the radial diffusion steady state solution for the low-energy particle population (Figure 8, middle) and the RCM simulation for the same energy electron fluxes (Figure 9, middle), the simulations using these two distributions for low-energy boundary conditions show similar results (Figure 8, bottom, and Figure 9, bottom). The fluxes of $1 \mathrm{MeV}$ equatorially mirroring particles, on Figure 9 (bottom), indicate the convective injection of the particles at $L^{*}=7$ in the initial phase of the storm, the inward radial motion of the fluxes during the main phase, and the buildup of the peak at $L^{*}=4$ during the recovery phase. In Figure 9 (bottom), the peak in fluxes is slightly more pronounced, which suggests that the fluxes are slightly underestimated in the simulation using the radial diffusion steady state solution as a low-energy boundary condition (Figure 8, bottom). The overall similarity of the results might suggest that the steady state solution of the radial diffusion equation can be an acceptable assumption for the low-energy boundary at $\mu=11 \mathrm{MeV} / \mathrm{G}$. Chorus waves cannot efficiently accelerate such low-energy electrons to $\mathrm{MeV}$ energies unless the electrons are also accelerated by the inward radial diffusion. This is consistent with the study by Horne et al. [2005], who showed that electrons with energies below $300 \mathrm{keV}$ are affected by strong losses, while electrons above $300 \mathrm{keV}$ can be further accelerated by chorus waves throughout a storm.

\section{4. “Open" Boundary}

[40] Our initial RCM simulation result presented in Figure 6 indicates that magnetospheric convection by itself may be insufficient to explain the observed relativistic electron fluxes at geosynchronous orbit. To study the origin of the relativistic electrons near the geosynchronous orbit, we allow the radiation belt electrons to diffuse outward to $L^{*}=7$ and change the $\mathrm{MeV}$ particle population at the outer $L^{*}$ boundary. To that end, we set up a zero gradient boundary condition instead of the constant value boundary condition as in the previous runs. This condition will be hereafter referred as an "open" boundary condition, since we allow diffusion to change values on the boundary. To account for the convective source of low-energy electrons, we add $\mathcal{S}_{\text {conv }}$ at $L^{*}=7$ in equation (1). This source is calculated as a difference between RCM results at the current time step and the previous time step

$$
\mathcal{S}_{\text {conv }}=\left.\frac{\partial f_{R C M}}{\partial t}\right|_{L^{*}=7},
$$

where $f_{R C M}$ is the PSD calculated by RCM.

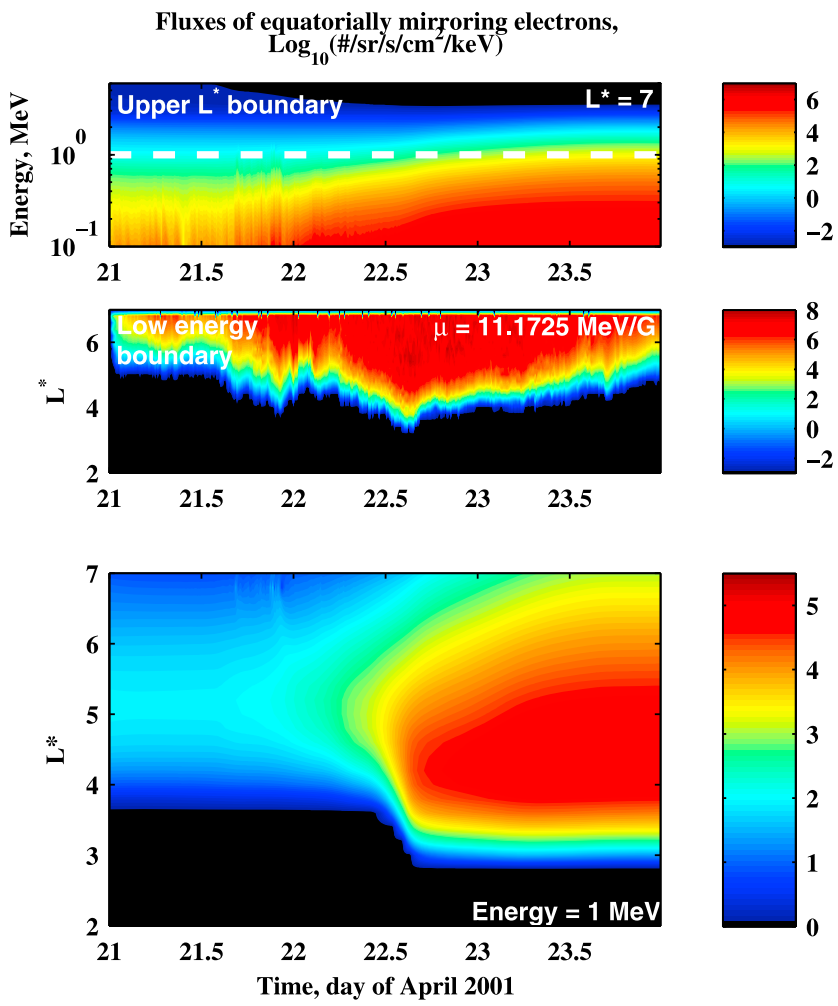

Figure 10. Same as Figure 9 but with an "open" outer $L^{*}$ boundary (see text for details). (top) Fluxes calculated by the VERB code at $L^{*}=7$. A dashed line is plotted at $1 \mathrm{MeV}$ energy. (middle) Fluxes calculated by the RCM code at $\mu=$ $11 \mathrm{MeV} / \mathrm{G}$ (low-energy boundary). (bottom) Electron fluxes of $1 \mathrm{MeV}$ equatorially mirroring electrons resulting from VERB simulation. Fluxes are color coded as $\log _{10}(\# / s r / s /$ $\mathrm{cm}^{2} / \mathrm{keV}$ ). 
[41] The results of this simulation are shown in Figure 10. Electron fluxes at $E=1 \mathrm{MeV}$ (Figure 10, bottom) clearly show an increased outward radial diffusion during the recovery phase of the storm. As compared to Figure 9 (top), the fluxes at $L^{*}=7$ (Figure 10, top) are much more constant. Figure 10 (top) also shows the increase of $1 \mathrm{MeV}$ fluxes at $L^{*}=7$, which are now more consistent with the statistically averaged data (Figure 7, top). In the simulation with the "open" outer $L^{*}$ boundary condition, outward radial diffusion transports electrons that were accelerated by energy diffusion to $\mathrm{MeV}$ energy in the heart of the radiation belts. The resulting fluxes have larger values as compared to these in Figure 7 because of the absence of losses to the magnetopause in the last simulation and the absence of possibly important losses due to a resonance interaction with EMIC waves.

\section{Conclusions}

[42] We performed a coupled simulation of the VERB code with the RCM code. The RCM code is capable of computing magnetospheric convection, while the VERB code is used for the radial, pitch angle, and energy diffusion due to resonant wave-particle interactions in the radiation belts. Magnetospheric convection, calculated by the RCM code, provided the seed population for the VERB code simulations. Coupling of the convective and diffusive magnetospheric processes allowed us to perform coupled time-dependent simulations of the radiation belt dynamics, and to make an initial assessment of the origin of relativistic electrons at geosynchronous orbit.

[43] We performed a simulation of the VERB code with RCM-calculated fluxes applied to the outer $L^{*}$ boundary and compared the results with a simulation using the timeindependent boundary conditions obtained from the statistically averaged data. Both simulations showed an increase of fluxes around $L^{*}=4$ in the recovery phase, while the coupled simulation showed a greater enhancement of the radiation belt electron fluxes as compared to the stand alone VERB simulation with time-independent boundary conditions. The comparison of simulations indicates the importance of using a realistic time-varying electron population at the outer $L^{*}$ boundary for radiation belt simulations.

[44] We also applied RCM calculated fluxes as the lowenergy boundary condition. The results agreed well with a simulation that used an assumption of balance between convective source and losses at the low-energy boundary, indicating that $\mathrm{MeV}$ fluxes are relatively insensitive to the assumed fluxes at low values of the first adiabatic invariant, and are mostly determined by the seed population at the outer $L^{*}$ boundary. The fluxes with energies lower than $\mathrm{MeV}$, however, can be more sensitive to the low-energy boundary since these fluxes are located closer to the boundary in phase space.

[45] To study the origin of the $\mathrm{MeV}$ electrons at geosynchronous orbit, we performed a coupled simulation with an "open" outer $L^{*}$ boundary condition, which allowed outward radial diffusion to change the particle population at $L^{*}=7$. The results of such a simulation indicated that it is possible for relativistic electrons to reach $L^{*}=7$ by outward radial diffusion combined with the energization of the lowenergy particles due to resonant wave-particle interactions at lower $\mathrm{L}$ shells. The idea that the origin of multi-MeV electrons at geosynchronous orbit is the result of outward radial diffusion has previously been proposed by several authors. Reeves et al. [1998] presented observations of electron fluxes during a January 1997 geomagnetic storm. Outward radial diffusion was proposed to be a potential process by which electrons are brought to higher L shells, after the electrons were accelerated within the radiation belts. While it was previously believed that electrons with energies of hundreds of $\mathrm{keV}$ form a seed population for MeV electrons, Varotsou et al. [2008] and Shprits et al. [2009], using 3-D modeling, further showed that electrons with energies as low as tens of $\mathrm{keV}$ can be accelerated by means of combined radial transport, conserving the first adiabatic invariant and local acceleration. Low-energy $(\sim$ tens of $\mathrm{keV}$ ) electrons are brought inside geosynchronous orbit with steady convection and radial diffusion. While diffusing to lower $L^{*}$, these electrons are also accelerated by chorus wave driven energy diffusion, which forms peaks in PSD around $L^{*}=4-5$. These peaks are smoothed out by inward radial diffusion, which brings electrons to a lower $L^{*}$, and by outward radial diffusion, which brings the majority of the $\mathrm{MeV}$ electrons to geosynchronous orbit.

[46] The presented work describes the initial results of the coupled convection-diffusion radiation belt simulation. A number of improvements would need to be made to the coupled model before these qualitative results can become quantitatively accurate enough to be verified with satellite observations. Among these improvements are the computation of a self-consistent magnetic field by the RCM, and the use of the RCM for the advective transport of energetic electrons inside $L^{*}=7$. The second would require that the RCM and VERB codes exchange information on the dynamics of the electron fluxes at each time step, i.e., a two-way coupling. Comparison of simulation results with observations will allow us to quantify the possible differences between observations and simulations, in order to identify important physical processes that might be missing from the models.

[47] The coupled RCM-VERB model is driven only by the solar wind parameters and $K p$ index and is independent of satellite observation, which is critical for radiation belt forecasting and space weather predictions.

\section{Appendix A: List of Important Assumptions}

[48] The coupled model describes a broad range of physical processes, which involves a list of assumptions. Here we present a list of the important assumptions made in the computation and coupling process. Although the relative importance of any particular assumption is arguable, we consider these assumptions to be those with the most important physical consequences.

[49] The list of important assumptions is as follows.

[50] 1. The magnetospheric plasma wave parameters are either not well known or are only known with a large uncertainty. The latitudinal distribution of chorus waves [Li et al., 2007] on the dayside has very high uncertainties, since it was based on CRRES measurements that did not provide complete MLT and latitudinal coverage. Geomagnetic dependence was derived for high-latitude dayside chorus waves [Shprits et al., 2007b] and was assumed to be similar for low-latitude nightside chorus waves. This might 
be not a good assumption [ $\mathrm{Li}$ et al., 2009]. Variations of the wave parameters can lead to significant changes in the diffusion coefficients and the simulation results [Shprits et al., 2006b].

[51] 2. RCM simulations assume that the pitch angle distribution of electrons is isotropic.

[52] 3. The RCM results were MLT averaged over particle's drift path.

[53] 4. The magnetic field in the RCM simulation was not computed self-consistently.

[54] 5. The energy spectrum used as a boundary condition at $L^{*}=7$ was obtained from the equatorial fluxes measured by the LANL and CRRES satellites and time averaged over a long time period [Shprits et al., 2009].

[55] 6. Cross energy-pitch angle and L-pitch angle terms were neglected.

[56] 7. To convert from PSD to fluxes and compare the fluxes with observations, a realistic magnetic field should be used to account for adiabatic variations that may be dominant in the outer region.

[57] 8. Other plasma waves (i.e., EMIC, magnetosonic waves) might play an important role in radiation belts dynamics.

[58] Acknowledgments. This research was supported by the NASA grant NNX09AF51G, NSF GEM grant ATM-0603191, AFRL grant FA9550-08-1-0140, and lab Research Fee grant 09-LR-04-200 116720SHPY. The work by Larry R. Lyons and M. Gkioulidou has been supported by NASA grants NNX07AF66G and NNX09AQ41H and NSF grant ATM0819864. The authors would like to thank Chih-Ping Wang for providing information of the boundary conditions used in the RCM simulation.

[59] Masaki Fujimoto thanks the reviewer for his or her assistance in evaluating this paper.

\section{References}

Albert, J. M. (2007), Simple approximations of quasi-linear diffusion coefficients, J. Geophys. Res., 112, A12202, doi:10.1029/2007JA012551.

Albert, J. M., and S. L. Young (2005), Multidimensional quasi-linear diffusion of radiation belt electrons, Geophys. Res. Lett., 32, L14110, doi:10.1029/2005GL023191.

Albert, J. M., N. P. Meredith, and R. B. Horne (2009), Three-dimensional diffusion simulation of outer radiation belt electrons during the 9 October 1990 magnetic storm, J. Geophys. Res., 114, A09214, doi:10.1029/ 2009JA014336.

Baker, D. N., and E. C. Stone (1978), The magnetopause energetic electron layer: 1. Observations along the distant magnetotail, J. Geophys. Res., 83(A9), 4327-4338, doi:10.1029/JA083iA09p04327.

Baker, D. N., J. H. Allen, and R. D. Belian (1996), An assessment of the space environmental conditions during the recent Anik E1 spacecraft operational failure, ISTP Newsl., 6(2), 8-29.

Beutier, T., and D. Boscher (1995), A 3-dimensional analysis of the electron-radiation belt by the Salammbo code, J. Geophys. Res., 100(A8), 14,853-14,861.

Boscher, D., S. Bourdarie, R. M. Thorne, and B. Abel (2000), Influence of the wave characteristics on the electron radiation belt distribution, J. Geophys. Res., 26(1), 163-166, doi:10.1029/2007JA012368.

Boscher, D., S. Bourdarie, P. O'Brien, and T. Guild (2008), ONERADESP library V4.2, Off. Natl. d'Etudes et de Rech. Aerosp., Toulouse, France.

Bourdarie, S., D. Boscher, T. Beutier, J. A. Sauvaud, and M. Blanc (1996), Magnetic storm modeling in the Earth's electron belt by the Salammbo code, J. Geophys. Res., 101(A12), 27,171-27,176.

Bourdarie, S., D. Boscher, T. Beutier, J. A. Sauvaud, and M. Blanc (1997), Electron and proton radiation belt dynamic simulations during storm periods: A new asymmetric convection-diffusion model, J. Geophys. Res., 102(A8), 17,541-17,552.

Brautigam, D. H., and J. M. Albert (2000), Radial diffusion analysis of outer radiation belt electrons during the October 9, 1990, magnetic storm, J. Geophys. Res., 105(A1), 291-309.

Carpenter, D., and R. Anderson (1992), An ISEE/whistler model of equatorial electron density in the magnetosphere, J. Geophys. Res., 97(A2), 1097-1108.
Craven, J. (1966), Temporal variations of electron intensities at low altitudes in the outer radiation zone as observed with satellite Injun 3 , J. Geophys. Res., 71(23), 5643-5663.

Elkington, S. R., M. Wiltberger, A. A. Chan, and D. N. Baker (2004) Physical models of the geospace radiation environment, J. Atmos. Sol. Terr. Phys., 66(15-16), 1371-1387, doi:10.1016/j.jastp.2004.03.023.

Falthamm, C., and M. Walt (1969), Radial motion resulting from pitchangle scattering of trapped electrons in distorted geomagnetic field, J. Geophys. Res., 74(16), 4184-4186.

Fok, M.-C., R. B. Horne, N. P. Meredith, and S. A. Glauert (2008), Radiation belt environment model: Application to space weather nowcasting, J. Geophys. Res., 113, A03S08, doi:10.1029/2007JA012558.

Gkioulidou, M., C.-P. Wang, L. R. Lyons, and R. A. Wolf (2009), Formation of the Harang reversal and its dependence on plasma sheet conditions: Rice Convection Model simulations, J. Geophys. Res., 114, A07204, doi:10.1029/2008JA013955.

Glauert, S. A., and R. B. Horne (2005), Calculation of pitch angle and energy diffusion coefficients with the PADIE code, J. Geophys. Res., 110, A04206, doi:10.1029/2004JA010851.

Horne, R. B., N. P. Meredith, R. M. Thorne, D. Heynderickx, R. H. A. Iles, and R. R. Anderson (2003), Evolution of energetic electron pitch angle distributions during storm time electron acceleration to megaelectronvolt energies, J. Geophys. Res., 108(A1), 1016, doi:10.1029/2001JA009165.

Horne, R. B., et al. (2005), Wave acceleration of electrons in the Van Allen radiation belts, Nature, 437, 227-230, doi:10.1038/nature03939.

Jacobs, J. A., Y. Kato, S. Matsushita, and V. A. Troitskaya (1964), Classification of geomagnetic micropulsations, J. Geophys. Res., 69(1), 180-181, doi:10.1029/JZ069i001p00180.

Jordanova, V. K., and Y. Miyoshi (2005), Relativistic model of ring current and radiation belt ions and electrons: Initial results, Geophys. Res. Lett., 32, L14104, doi:10.1029/2005GL023020.

Kennel, C. F., and F. Engelmann (1966), Velocity space diffusion from weak plasma turbulence in a magnetic field, Phys. Fluids, 9, 2377.

Kennel, C. F., and H. E. Petschek (1966), Limit on stably trapped particle fluxes, J. Geophys. Res., 71(1), 1-28, doi:10.1029/JZ071i001p00001.

Lanzerotti, L. J. (2001), Space weather effects on technologies, Space Weather, 125, 11-22.

Lenchek, A., S. Singer, and R. Wentworth (1961), Geomagnetically trapped electrons from cosmic ray albedo neutrons, J. Geophys. Res. 66(12), 4027-4046, doi:10.1029/JZ066i012p04027.

Lerche, I. (1968), Quasilinear theory of resonant diffusion in a magnetoactive, relativistic plasma, Phys. Fluids, $11,1720$.

Li, W., Y. Y. Shprits, and R. M. Thorne (2007), Dynamic evolution of energetic outer zone electrons due to wave-particle interactions during storms, J. Geophys. Res., 112, A10220, doi:10.1029/2007JA012368.

Li, W., et al. (2009), Global distribution of whistler-mode chorus waves observed on the THEMIS spacecraft, Geophys. Res. Lett., 36, L09104, doi:10.1029/2009GL037595.

Li, W., R. M. Thorne, J. Bortnik, Y. Nishimura, V. Angelopoulos, L. Chen, J. P. McFadden, and J. W. Bonnell (2010), Global distributions of suprathermal electrons observed on THEMIS and potential mechanisms for access into the plasmasphere, J. Geophys. Res., 115, A00J10, doi:10.1029/2010JA015687.

Liu, S., M. Chen, L. Lyons, H. Korth, J. Albert, J. Roeder, P. Anderson, and M. Thomsen (2003), Contribution of convective transport to stormtime ring current electron injection, J. Geophys. Res., 108(A10), 1372, doi:10.1029/2003JA010004.

Lyons, L. R. (1984), Electron energization in the geomagnetic tail current sheet, J. Geophys. Res., 89(A7), 5479-5487, doi:10.1029/ JA089iA07p05479.

Lyons, L. R., and R. M. Thorne (1973), Equilibrium structure of radiation belt electrons, J. Geophys. Res., 78(13), 2142-2149.

Lyons, L. R., R. M. Thorne, and C. F. Kennel (1971), Electron pitch-angle diffusion driven by oblique whistler-mode turbulence, J. Plasma Phys., 6, 589-606.

Lyons, L., R. Thorne, and C. Kennel (1972), Pitch-angle diffusion of radiation belt electrons within the plasmasphere, J. Geophys. Res., 77(19), 3455-3474.

Miyoshi, Y., A. Morioka, T. Obara, H. Misawa, T. Nagai, and Y. Kasahara (2003), Rebuilding process of the outer radiation belt during the 3 November 1993 magnetic storm: NOAA and Exos-D observations, J. Geophys. Res., 108(A1), 1004, doi:10.1029/2001JA007542.

Miyoshi, Y. S., V. K. Jordanova, A. Morioka, M. F. Thomsen, G. D. Reeves, D. S. Evans, and J. C. Green (2006), Observations and modeling of energetic electron dynamics during the October 2001 storm, J. Geophys. Res., 111, A11S02, doi:10.1029/2005JA011351.

Ni, B., R. M. Thorne, Y. Y. Shprits, and J. Bortnik (2008), Resonant scattering of plasma sheet electrons by whistler-mode chorus: Contribution 
to diffuse auroral precipitation, Geophys. Res. Lett., 35, L11106, doi:10.1029/ 2008GL034032.

Ni, B., R. M. Thorne, R. B. Horne, N. P. Meredith, Y. Y. Shprits, L. Chen, and W. Li (2011a), Resonant scattering of plasma sheet electrons leading to diffuse auroral precipitation: 1. Evaluation for electrostatic electron cyclotron harmonic waves, J. Geophys. Res., 116, A04218, doi:10.1029/ 2010JA016232.

Ni, B., R. M. Thorne, N. P. Meredith, R. B. Horne, and Y. Y. Shprits (2011b), Resonant scattering of plasma sheet electrons leading to diffuse auroral precipitation: 2. Evaluation for whistler mode chorus waves, J. Geophys. Res., 116, A04219, doi:10.1029/2010JA016233.

Odenwald, S., J. Greenb, and W. Taylor (2006), Forecasting the impact of an 1859-calibre superstorm on satellite resources, Adv. Space Res., 38(2), 280-297, doi:10.1016/j.asr.2005.10.046.

Orlova, K. G., and Y. Y. Shprits (2010), Dependence of pitch-angle scattering rates and loss timescales on the magnetic field model, Geophys. Res. Lett., 37, L05105, doi:10.1029/2009GL041639.

Reeves, G. D., D. N. Baker, R. D. Belian, J. B. Blake, T. E. Cayton, J. F Fennell, R. H. W. Friedel, M. M. Meier, R. S. Selesnick, and H. E. Spence (1998), The global response of relativistic radiation belt electrons to the January 1997 magnetic cloud, Geophys. Res. Lett., 25(17), 3265-3268, doi:10.1029/98GL02509.

Roederer, J., and M. Schulz (1969), Effect of shell splitting on radial diffusion in the magnetosphere, J. Geophys. Res., 74(16), 4117-4122.

Roederer, J., and M. Schulz (1971), Splitting of drift shells by the magnetospheric electric field, J. Geophys. Res., 76(4), 1055-1059.

Rothwell, P., and C. E. McIlwain (1960), Magnetic storms and the Van Allen radiation belts-Observations from satellite $1958 \varepsilon$ (Explorer IV), J. Geophys. Res., 65(3), 799-806.

Schulz, M. (1972), Drift-shell splitting at arbitrary pitch angle, J. Geophys. Res., 77(4), 624-634.

Schulz, M., and L. J. Lanzerotti (1974), Particle Diffusion in the Radiation Belts, Springer, Berlin.

Sheeley, B., M. Moldwin, H. Rassoul, and R. Anderson (2001), An empirical plasmasphere and trough density model: CRRES observations, J. Geophys. Res., 106(A11), 25,631-25,641.

Shprits, Y. Y., and B. Ni (2009), Dependence of the quasi-linear scattering rates on the wave normal distribution of chorus waves, J. Geophys. Res., 114, A11205, doi:10.1029/2009JA014223.

Shprits, Y. Y., and R. M. Thorne (2004), Time dependent radial diffusion modeling of relativistic electrons with realistic loss rates, Geophys. Res. Lett., 31, L08805, doi:10.1029/2004GL019591.

Shprits, Y. Y., R. M. Thorne, R. Friedel, G. D. Reeves, J. Fennell, D. N Baker, and S. G. Kanekal (2006a), Outward radial diffusion driven by losses at magnetopause, J. Geophys. Res., 111, A11214, doi:10.1029/ 2006JA011657.

Shprits, Y. Y., R. M. Thorne, R. B. Horne, and D. Summers (2006b) Bounce-averaged diffusion coefficients for field-aligned chorus waves, J. Geophys. Res., 111, A10225, doi:10.1029/2006JA011725.

Shprits, Y. Y., W. Li, and R. M. Thorne (2007a), Controlling effect of the pitch angle scattering rates near the edge of the loss cone on electron lifetimes, J. Geophys. Res., 111, A12206, doi:10.1029/2006JA011758.

Shprits, Y. Y., N. P. Meredith, and R. M. Thorne (2007b), Parameterization of radiation belt electron loss timescales due to interactions with chorus waves, Geophys. Res. Lett., 34, L11110, doi:10.1029/2006GL029050.

Shprits, Y. Y., D. A. Subbotin, and B. Ni (2009), Evolution of electron fluxes in the outer radiation belt computed with the VERB code, J. Geophys Res., 114, A11209, doi:10.1029/2008JA013784.

Subbotin, D. A., and Y. Y. Shprits (2009), Three-dimensional modeling of the radiation belts using the versatile electron radiation belt (VERB) code, Space Weather, 7, S10001, doi:10.1029/2008SW000452.

Subbotin, D. A., Y. Y. Shprits, and B. Ni (2010), Three-dimensional VERB radiation belt simulations including mixed diffusion, J. Geophys. Res., 115, A03205, doi:10.1029/2009JA015070.

Tao, X., A. A. Chan, J. M. Albert, and J. A. Miller (2008), Stochastic modeling of multidimensional diffusion in the radiation belts, J. Geophys. Res., 113, A07212, doi:10.1029/2007JA012985.

Tao, X., J. M. Albert, and A. A. Chan (2009), Numerical modeling of multidimensional diffusion in the radiation belts using layer methods, J. Geophys. Res., 114, A02215, doi:10.1029/2008JA013826.
Thorne, R. M., Y. Y. Shprits, N. P. Meredith, R. B. Horne, W. Li, and L. R. Lyons (2007), Refilling of the slot region between the inner and outer electron radiation belts during geomagnetic storms, J. Geophys. Res., 112, A06203, doi:10.1029/2006JA012176.

Thorne, R. M., B. Ni, X. Tao, R. B. Horne, and N. P. Meredith (2010), Scattering by chorus waves as the dominant cause of diffuse auroral precipitation, Nature, 467, 943-946, doi:10.1038/nature09467.

Toffoletto, F., S. Sazykin, R. Spiro, and R. Wolf (2003), Inner magnetospheric modeling with the Rice Convection Model, Space Sci. Rev. 107(1-2), 175-196, doi:10.1023/A:1025532008047.

Tsyganenko, N. A. (1989), A magnetospheric magnetic field model with a warped tail current sheet, Planet. Space Sci., 37, 5-20.

Tsyganenko, N. (1995), Modeling the Earth's magnetospheric magneticfield confined within a realistic magnetopause, J. Geophys. Res., 100(A4), 5599-5612.

Tsyganenko, N., and M. Sitnov (2005), Modeling the dynamics of the inner magnetosphere during strong geomagnetic storms, J. Geophys. Res., 110 A03208, doi:10.1029/2004JA010798.

Van Allen, J. A. (1959), Radiation belts around the Earth, Sci. Am., 200(3), 39-47.

Van Allen, J. A., and L. A. Frank (1959), Radiation around the Earth to a radial distance of 107,400 km, Nature, 183, 430-434.

Varotsou, A., D. Boscher, S. Bourdarie, R. B. Horne, S. A. Glauert, and N. P. Meredith (2005), Simulation of the outer radiation belt electrons near geosynchronous orbit including both radial diffusion and resonant interaction with whistler-mode chorus waves, Geophys. Res. Lett., 32, L19106, doi:10.1029/2005GL023282.

Varotsou, A., D. Boscher, S. Bourdarie, R. B. Horne, N. P. Meredith, S. A. Glauert, and R. H. Friedel (2008), Three-dimensional test simulations of the outer radiation belt electron dynamics including electron-chorus resonant interactions, J. Geophys. Res., 113, A12212, doi:10.1029/ 2007JA012862.

Vernov, S. N., and A. E. Chudakov (1960), Investigation of radiation in outer space, in Proceedings of the Moscow Cosmic Ray Conference, vol. 111, edited by S. I. Syrovatsky, pp. 19-29, Int. Union of Pure and Appl. Phys., Moscow.

Wang, C.-P., L. R. Lyons, T. Nagai, J. M. Weygand, and R. W. McEntire (2007), Sources, transport, and distributions of plasma sheet ions and electrons and dependences on interplanetary parameters under northward interplanetary magnetic field, J. Geophys. Res., 112, A10224, doi:10.1029/2007JA012522.

Weimer, D. (2001), An improved model of ionospheric electric potentials including substorm perturbations and application to the Geospace Environment Modeling November 24, 1996, event, J. Geophys. Res., 106(A1), $407-416$.

Xiao, F., Z. Su, H. Zheng, and S. Wang (2009), Modeling of outer radiation belt electrons by multidimensional diffusion process, J. Geophys. Res. 114, A03201, doi:10.1029/2008JA013580.

Xiao, F., Z. Su, H. Zheng, and S. Wang (2010), Three-dimensional simulations of outer radiation belt electron dynamics including cross diffusion terms, J. Geophys. Res., 115, A05216, doi:10.1029/2009JA014541.

M. Gkioulidou, L. R. Lyons, B. Ni, Y. Y. Shprits, D. A. Subbotin, and R. M. Thorne, Department of Atmospheric and Oceanic Sciences, University of California, 405 Hilgard Ave., 7127 Math Sciences Bldg., Los Angeles, CA 90095-1565, USA. (mgioul@atmos.ucla.edu; larry@atmos.ucla.edu; bbni@atmos.ucla.edu; yshprits@atmos.ucla.edu; subbotin@atmos.ucla. edu;rmt@atmos.ucla.edu)

R. B. Horne, British Antarctic Survey, Natural Environment Research Council, Madingley Road, High Cross, Cambridge CB3 0ET, UK. (rh@ bas.ac.uk)

M. K. Hudson, Department of Physics and Astronomy, Dartmouth College, 6127 Wilder Laboratory, Hanover, NH 03755, USA. (mary.k. hudson@dartmouth.edu)

V. G. Merkin, Johns Hopkins University Applied Physics Laboratory, Laurel, MD 20723, USA. (slava.merkin@jhuapl.edu)

F. R. Toffoletto, Department of Space Physics and Astronomy, William Marsh Rice University, MS 108, 6100 Main St., Houston, TX 77005, USA. (toffo@rice.edu) 